\title{
Status of a Search for Direct CP Violation in Hyperon Decays
}

\section{E. Craig Dukes* ${ }^{\dagger}$}

High Energy Physics Laboratory, University of Virginia, Charlottesville, VA, 22901, USA

E-mail: icraigdukes@virginia.edui

Abstract: The Fermilab HyperCP collaboration is making precision studies of charged hyperon and kaon decays. The primary goal of the experiment is a sensitive search for $C P$ violation in hyperon decays. The search is done by comparing the proton angular distribution in $\Xi^{-} \rightarrow \Lambda \pi^{-}, \Lambda \rightarrow p \pi^{-}$decays to that of the conjugate decays, the $C P$ violating observable being $A_{\Xi \Lambda}=\left(\alpha_{\Xi} \alpha_{\Lambda}-\alpha_{\bar{\Xi}} \alpha_{\bar{\Lambda}}\right) /\left(\alpha_{\Xi} \alpha_{\Lambda}+\alpha_{\bar{\Xi}} \alpha_{\bar{\Lambda}}\right)$. A brief status of the analysis is presented: preliminary studies indicate that at the level of few times $10^{-3}$ there is no asymmetry, the ultimate goal of the experiment being a sensitivity of $2 \times 10^{-4}$.

\section{Introduction}

In the standard model $C P$ violation lies with the complex phase in the Cabbibo-KobayashiMaskawa (CKM) matrix, the origin of which remains a mystery. Hence $C P$ violation should be a ubiquitous property of the weak interaction, and not just confined to kaon and beauty meson decays. A promising arena in which to look elsewhere for $C P$ violation is in the nonleptonic decays of hyperons, the asymmetry expected to be relatively large, as well as being sensitive to potential sources of $C P$ violation that, for example, $\epsilon^{\prime} / \epsilon$ is not. This is the goal of HyperCP, the first dedicated hyperon $C P$ violation experiment, which is probing hyperon $C P$ violation through a comparison of the angular distribution of the decays: $\Xi^{-} \rightarrow \Lambda \pi^{-}, \Lambda \rightarrow p \pi^{-}$and $\bar{\Xi}^{+} \rightarrow \bar{\Lambda} \pi^{+}, \bar{\Lambda} \rightarrow \bar{p} \pi^{+}$.

\footnotetext{
${ }^{*}$ Speaker.

${ }^{\dagger}$ Representing the HyperCP collaboration: A. Chan, Y.C. Chen, C. Ho, P.K. Teng, (Academia Sinica); W.S. Choong, G. Gidal, Y. Fu, P. Gu, T. Jones, K.B. Luk, B. Turko, P. Zyla, (Berkeley and LBNL); C. James, J. Volk, (FNAL); J. Felix, (Guanajuato); R.A. Burnstein, A. Chakravorty, D.M. Kaplan, L.M. Lederman, W. Luebke, D. Rajaram, H.A. Rubin, N. Solomey, Y. Torun, C.G. White, S.L. White, (IIT, Chicago); N. Leros, J.P. Perroud, (Lausanne); H.R. Gustafson, M.J. Longo, F. Lopez, H.K. Park, (Michigan); K. Clark, M. Jenkins, (S. Alabama); E.C. Dukes, C. Durandet, R. Godang, T. Holmstrom, M. Huang, L. Lu, K.S. Nelson, (Virginia).
} 


\section{Phenomenology of Hyperon $C P$ Violation}

The decay distribution of the daughter proton in the rest frame of the $\Lambda$ is anisotropic:

$$
\frac{\mathrm{dN}}{d \Omega}=\frac{\mathrm{N}_{0}}{4 \pi}\left(1+\alpha_{\Lambda} \vec{P}_{\Lambda} \cdot \hat{p}_{p}\right)
$$

where $\vec{P}_{\Lambda}$ is the $\Lambda$ polarization, $\hat{p}_{p}$ is the proton momentum direction in the rest frame of the $\Lambda$, and $\alpha_{\Lambda}$ is, in terms of the $S$ - and $P$-wave amplitudes:

$$
\alpha_{\Lambda}=\frac{2 \operatorname{Re}\left(S^{*} P\right)}{|S|^{2}+|P|^{2}}
$$

If $C P$ is conserved then $\alpha_{\Lambda}$ transforms according to $\alpha_{\Lambda}=-\alpha_{\bar{\Lambda}}$ and hence an observable sensitive to $C P$ violation is:

$$
A_{\Lambda}=\frac{\alpha_{\Lambda}+\alpha_{\bar{\Lambda}}}{\alpha_{\Lambda}-\alpha_{\bar{\Lambda}}}
$$

As one can see from Eq. $\overline{2} .1_{3}^{1}$ determining $\alpha_{\Lambda}$ requires precise knowledge of the parent $\Lambda$ polarization. This is done in Hyper $C P$ by requiring that the $\Lambda$ come from the decay of an unpolarized $\Xi$ hyperon: $\Xi \rightarrow \Lambda \pi$, whence $P_{\Lambda}=\alpha_{\Xi}$. The $\Xi$ hyperons are made with zero polarization by requiring that they be produced at zero degrees, where parity conservation in the strong interaction requires that the polarization be identically zero. If $C P$ symmetry is good in both $\Xi$ and $\Lambda$ decays, the decay distributions of the proton and anti-proton are identical in that $\Lambda$ rest frame - the $\Lambda$ helicity frame - in which the $\Lambda$ direction in the $\Xi$ rest frame defines the polar axis:

$$
\frac{d \mathrm{~N}}{d(\cos \theta)}=\frac{\mathrm{N}_{0}}{2}\left(1+\alpha_{\Lambda} P_{\Lambda} \cos \theta\right)=\frac{\mathrm{N}_{0}}{2}\left(1+\alpha_{\Lambda} \alpha_{\Xi} \cos \theta\right)=\frac{\mathrm{N}_{0}}{2}\left(1+\alpha_{\bar{\Lambda}} \alpha_{\bar{\Xi}} \cos \theta\right) .
$$

It is evident from the above equation that differences between the slopes of the $\Xi^{-}$and $\bar{\Xi}^{+}$ $\cos \theta$ distributions can be due to $C P$ violation in either the $\Xi$ or $\Lambda$ decays - the experiment is by necessity sensitive to $C P$ violation in both:

$$
A_{\Xi \Lambda}=\frac{\alpha_{\Xi} \alpha_{\Lambda}-\alpha_{\Xi} \alpha_{\bar{\Lambda}}}{\alpha_{\Xi} \alpha_{\Lambda}+\alpha_{\Xi} \alpha_{\bar{\Lambda}}} \cong A_{\Xi}+A_{\Lambda} .
$$

\section{Expectations}

The $C P$ asymmetry is manifested through the interference of the $S$ - and $P$-wave amplitudes, with a difference in the $S$ - and $P$-wave final-state strong-interaction phases needed for $\alpha_{\Lambda}$ or $\alpha_{\Xi}$ to be non-zero. The $p \pi^{-}$hadronic phase shifts have been measured to be: $\delta^{P}-\delta^{S}=7.1^{\circ}$ with an error of about $\pm 1.5^{\circ}$ [i] shifts, but recent calculations using chiral perturbation theory give values consistent with zero [2], implying that the contribution of $A_{\Xi}$ to $A_{\Xi \Lambda}$ will be small.

Calculations of the $C P$ asymmetries in hyperon decays are difficult and results are not thought to be reliable to better than an order of magnitude, the largest uncertainty being the determination of the hadronic matrix elements. Standard model predictions of $A_{\Xi \Lambda}$ range from about $10^{-4}$ to $10^{-5}$. Note that because $A_{\Xi \Lambda}$ probes both parity conserving and 
parity violating $C P$ amplitudes, it is sensitive to potential sources of $C P$ violation that $\epsilon^{\prime} / \epsilon$ is not. For example, some supersymmetric models that do not generate $\epsilon^{\prime} / \epsilon$ can lead to $A_{\Lambda}$ of $O\left(10^{-3}\right)\left[\begin{array}{l}3 \\ 0\end{array}\right]$.

Two experiments have studied hyperon $C P$ violation with roughly $1-2 \%$ precision. At LEAR the PS185 experiment measured $A_{\Lambda}=0.013 \pm 0.022$ [in], and at Fermilab the E756 experiment measured $A_{\Xi \Lambda}=0.012 \pm 0.014$ [ Neither of these measurements is sensitive enough to confront theoretical predictions and both are limited by statistical and not systematic errors.

\section{The HyperCP Experiment}

A plan view of the HyperCP spectrometer is shown in Fig. The apparatus consisted of a target immediately followed by a curved collimator — with a $4.88 \mu$ sr solid angle acceptance - embedded in a $6 \mathrm{~m}$ long dipole magnet. Charged particles were deflected up at a mean angle of $19.5 \mathrm{mrad}$. Following a vacuum decay region was a conventional magnetic spectrometer employing high-rate, narrow-pitch wire chambers. At the rear of the spectrometer was a muon system used for the study of rare and forbidden hyperon decays. A simple, yet selective trigger was formed by requiring the coincidence at the rear of the spectrometer of charged particles on either side of the spectrometer. Scintillator hodoscopes on the pion and proton sides and a hadronic calorimeter (on the proton side) were used to detect the charged particles. The calorimeter suppressed triggers from secondary-beam interactions with spectrometer material.

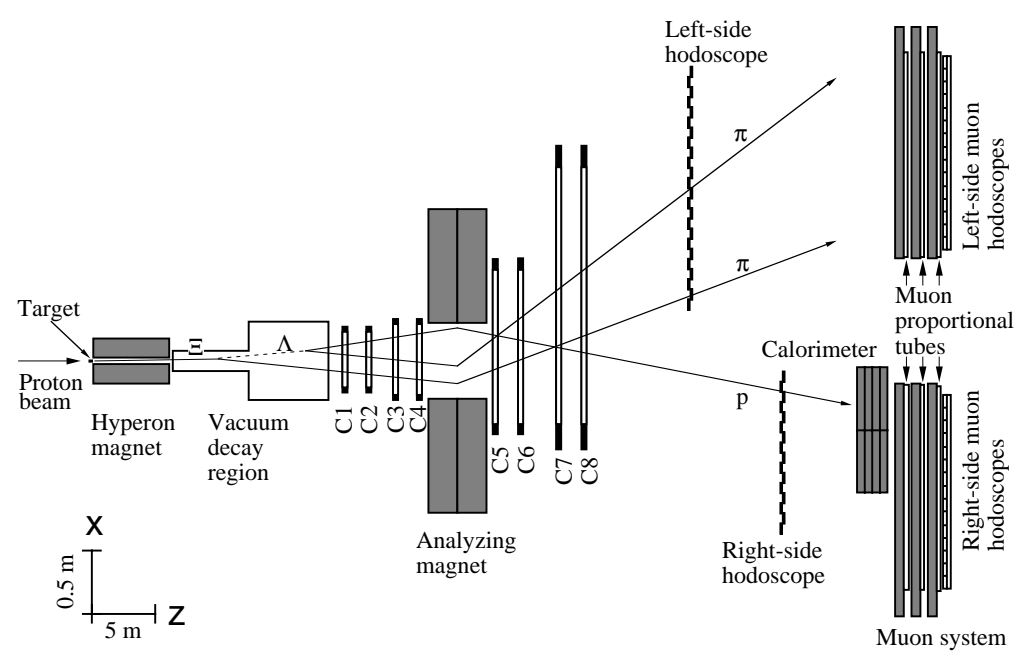

Figure 1: Plan view of the Hyper $C^{P}$ spectrometer.

With $800-\mathrm{GeV} / \mathrm{c}$ protons incident on a $2 \times 2 \mathrm{~mm}^{2} \mathrm{Cu}$ target, the mean momentum acceptance of the magnetic channel was about $170 \mathrm{GeV} / \mathrm{c}$. The polarities of both the Hyperon and Spectrometer magnets were periodically reversed to change from $\Xi^{-}$to $\bar{\Xi}^{+}$ running. A typical primary beam intensity of $7.5 \times 10^{9}$ protons per second gave a secondary beam rate of $13 \mathrm{MHz}$ at the exit of the collimator. 


\section{Status of the Analysis}

Space limitations preclude anything but a brief status of the analysis. A total of 231 billion triggers were written on 29401 tapes in two runs: 1997 and 1999 (see Table $i_{1}^{1}$ ), quite possibly the largest number of events ever recorded by a particle physics experiment. Much of 2000-2001 was spent in reconstructing the data on the Fermilab computer farms, yielding a total of 2.49 billion $\Xi^{-}$and $\bar{\Xi}^{+}$events. This translates to a sensitivity in $A_{\Xi \Lambda}$ of about $2 \times 10^{-4}$. The positive and negative data are very well matched, as can be seen from the $p \pi \pi$ invariant mass plot from all of the farmed data shown in Fig. ${ }_{2}^{2}$

The measurement of $\alpha_{\Lambda} \alpha_{\Xi}$ is done by the measurement of the slope of the proton distribution in the $\Lambda$ helicity frame. It is important to emphasize that since the polar axis of the $\Lambda$ helicity frame - defined by the $\Lambda$ direction in the $\Xi$ rest frame changes from event to event, acceptance differences localized to a particular part of the apparatus do not map to a particular part of the proton $\cos \theta$ distribution, greatly reducing potential sources of bias.

\begin{tabular}{lccc}
\hline \hline & \multicolumn{2}{c}{ Run } \\
& 1997 & 1999 & Total \\
\hline Tapes & 8980 & 20421 & 29401 \\
Data (TB) & 38 & 82 & 120 \\
Triggers $\left(10^{9}\right)$ & 58 & 173 & 231 \\
\hline \multicolumn{4}{c}{ Polarity } \\
& + & - & Total \\
\hline$\Xi$ events $\left(10^{9}\right)$ & 0.458 & 2.032 & 2.490 \\
\hline \hline
\end{tabular}

Table 1: Summary of data taken.

Two different measurements of $A_{\Xi \Lambda}$ are being done. Both have to deal with a data sample of such size that normal analysis tools and techniques cannot be applied.

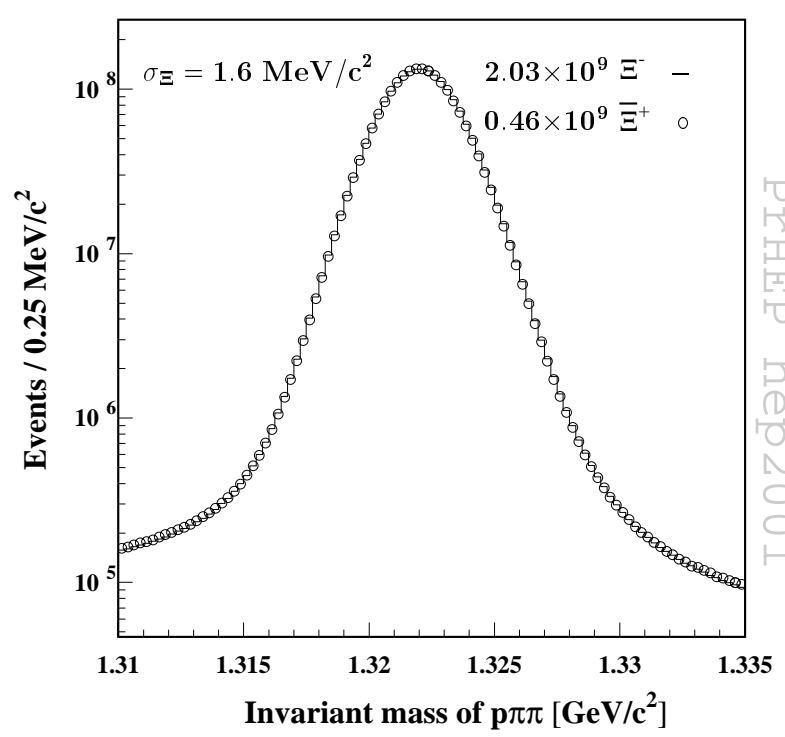

Figure 2: The $p \pi \pi$ invariant mass from the primary event reconstruction on the Fermilab farms. Only loose cuts have been applied.

The first technique uses a hybrid Monte Carlo (HMC) to measure the acceptance of the spectrometer. This is done by taking a real $\Xi \rightarrow \Lambda \pi, \Lambda \rightarrow p \pi$ event, discarding the proton and pion from the $\Lambda$ decay, and generating ten unpolarized $\Lambda$ decays. The proton and pion are traced through a model of the apparatus and a partial event reconstruction is performed at a level sufficient to determine whether the event would have been fully reconstructed. The HMC proton $\cos \theta$ distribution is parameterized in terms of a polynomial in $\alpha_{\Lambda} \alpha_{\Xi}$, with $\alpha_{\Lambda} \alpha_{\Xi}$ being determined by minimizing the difference between the real and HMC proton $\cos \theta$ distributions. Both $\alpha_{\Lambda} \alpha_{\Xi}$ and $\alpha_{\bar{\Lambda}} \alpha_{\bar{\Xi}}$ are then compared to search for any $C P$ asymmetry.

The second technique makes no attempt to measure the apparatus acceptance and requires no MC event generation, but rather directly compares the $\Xi^{-}$and $\bar{\Xi}^{+}$proton $\cos \theta$ 
distributions without acceptance corrections. The differences between the $\Xi^{-}$and $\bar{\Xi}^{+}$data samples are minimized by first binning both the data samples in the five-dimensional space of: the momentum of the $\Xi$, the two transverse coordinates of the $\Xi$ at the target, and the two transverse coordinates of the $\Xi$ at the exit of the collimator. The number of $\Xi^{-}$and $\bar{\Xi}^{+}$events in each bin is then made equal by weighting each cell by the ratio of the number of $\bar{\Xi}^{+}$to the number of $\Xi^{-}$events found in that cell.

A test of this method was done on a small sample (1.7\%) of the 1997 data [i6]. To demonstrate that the weighting technique does not kill a hypothetical $C P$ signal, two samples of $20 \times 10^{6} \bar{\Xi}^{+}$and $150 \times 10^{6} \Xi^{-}$MC events, each with $\Xi$ momentum distributions based on real data, were generated with $A_{\Xi \Lambda}=-17.2 \times 10^{-3}$. The comparison of the proton and antiproton $\cos \theta$ distributions after weighting was consistent with the input value with $A_{\Xi \Lambda}=(-16.9 \pm 0.4) \times 10^{-3}$.

A preliminary analysis was done on a sample of $5.9 \times 10^{6} \bar{\Xi}^{+}$and $37.2 \times 10^{6} \Xi^{-}$ events from the 1997 data. This analysis included a study of sources of systematic errors including: differences in the $x$ and $y$ displacements at the target, the effect of interactions in the spectrometer material, backgrounds, and the weighting method itself. The data were divided up into four sets. Figure tiproton to proton $\cos \theta$ distributions for one of those sets before and after weighting. The resulting $C P$-asymmetry was found to be consistent with zero:

$A_{\Xi \Lambda}=[-1.6 \pm 1.3$ (stat) \pm 1.1 (syst) $] \times 10^{-3}$.

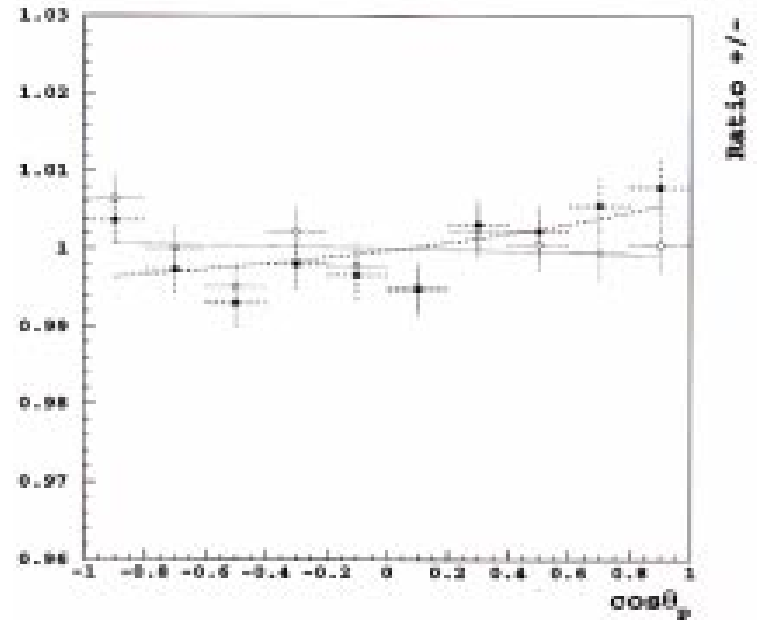

Figure 3: The ratio of the antiproton to proton $\cos \theta$ distributions in the $\Lambda$-helicity frame. Closed (open) diamonds are before (after) weighting.

The systematic error of this preliminary study is only an upper value limited by statistics. With the completion of the reconstruction of the entire data sample, high-statistics studies of the systematic errors are underway. We expect a total systematic error on the order of our estimated statistical precision, or about $10^{-4}$.

\section{References}

[1] L.D. Roper, R.M. Wright, and B.T. Feld, Phys. Rev. 138 1965$)$ B190.'

[2] M. Lu, M.B. Wise, and M.J. Savage, 'P 2 hys. Lett. B $3 \overline{3} \overline{7}(1 \overline{9} \overline{4})$ 133i.

[3] X.-G. He, H. Hurayama, S. Pakvasa, and G. Valencia, Phys. Rev. D'61 $(2000) 071701(\bar{R})_{1}^{\prime}$

[4] P.D. Barnes et al., 'Phys. Rev. $\mathbf{C} \mathbf{5 4}(1996) 1877$.

[5] K.B. Luk at al., iPhys. Rev. Lett. 85 (2000) $48 \overline{6} 60$.

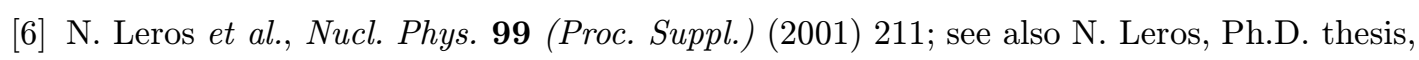
Univ. Lausanne, 2001 (unpublished). 